EPJ Web of Conferences 62, 06001 (2013)

DOI: $10.1051 /$ epjconf/20136206001

(C) Owned by the authors, published by EDP Sciences, 2013

\title{
Hidden systematics of fission channels
}

\author{
Karl-Heinz Schmidt ${ }^{\mathrm{a}}$ and Beatriz Jurado \\ CENBG, CNRS/IN2P3, Chemin du Solarium, BP. 120, 33175 Gradignan, France
}

\begin{abstract}
It is a common procedure to describe the fission-fragment mass distributions of fissioning systems in the actinide region by a sum of at least 5 Gaussian curves, one for the symmetric component and a few additional ones, together with their complementary parts, for the asymmetric components. These components have been attributed to the influence of fragment shells, e.g. in the statistical scission-point model of Wilkins, Steinberg and Chasman. They have also been associated with valleys in the potential-energy landscape between the outer saddle and the scission configuration in the multi-channel fission model of Brosa. When the relative yields, the widths and the mean mass-asymmetry values of these components are fitted to experimental data, the mass distributions can be very well reproduced. Moreover, these fission channels are characterised by specific values of charge polarisation, total kinetic energy and prompt-neutron yields. The present contribution investigates the systematic variation of the characteristic fission-channel properties as a function of the composition and the excitation energy of the fissioning system. The mean position of the asymmetric fission channels in the heavy fragment is almost constant in atomic number. The deformation of the nascent fragments at scission, which is the main source of excitation energy of the separated fission fragments ending up in prompt-neutron emission, is found to be a unique function of $Z$ for the light and the heavy fragment of the asymmetric fission channels. A variation of the initial excitation energy of the fissioning system above the fission saddle is only seen in the neutron yield of the heavy fragment. The charge polarisation in the two most important asymmetric fission channels is found to be constant and to appreciably exceed the macroscopic value. The variation of the relative yields and of the positions of the fission channels as a function of the composition and excitation energy of the fissioning system obey a hidden systematics that can be explained by the number of states in the vicinity of the outer fission barrier as a function of mass asymmetry, if the potential is constructed as the sum of the macroscopic contribution of the compound nucleus and empirically determined fragment shells. This hidden systematics also explains the transition from asymmetric to symmetric fission around ${ }^{226} \mathrm{Th}$ and around ${ }^{258} \mathrm{Fm}$.
\end{abstract}

\section{Introduction}

In the theory of fission, important progress has been made. The potential-energy surface of the fissioning systems has been systematically mapped with shell-model calculations in five-dimensional deformation space [1]. Stochastic methods [2-4] and self-consistent microscopic approaches [5] have

\footnotetext{
a e-mail: schmidt-erzhausen@t-online.de
}

This is an Open Access article distributed under the terms of the Creative Commons Attribution License 2.0, which permits unrestricted use, distribution, and reproduction in any medium, provided the original work is properly cited. 


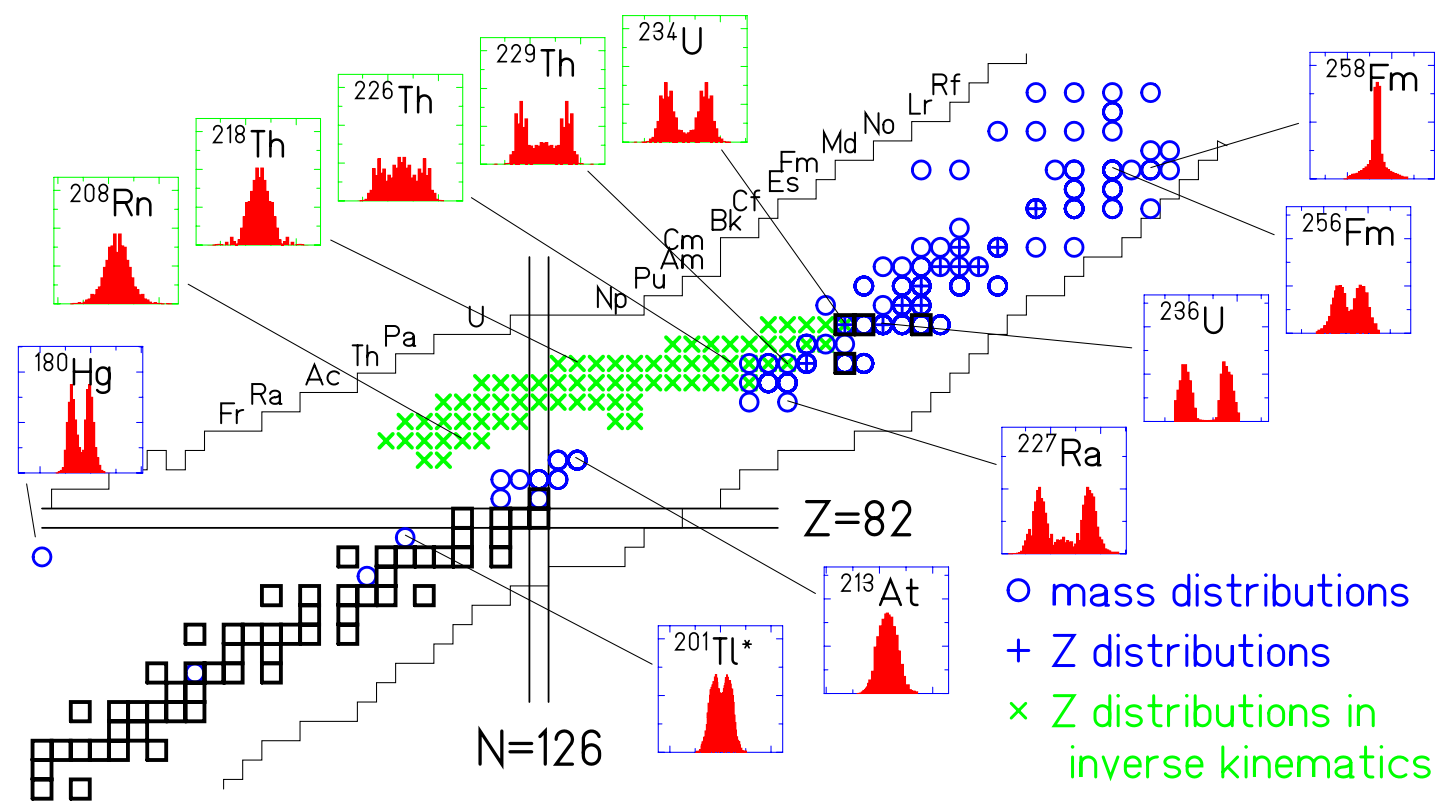

Figure 1. General view on the systems for which mass or nuclear-charge distributions have been measured. The distributions are shown for 12 selected systems. Blue circles (blue crosses): Mass (nuclear-charge) distributions, measured in conventional experiments [8, 9] and references given in [7]. Green crosses: Nuclear-charge distributions, measured in inverse kinematics [7].

been developed for dynamical calculations of low-energy fission. However, these calculations still face severe restrictions that are caused by the lack of suitable theoretical formalisms. The theoretical understanding of the fission process cannot fully rely on the powerful standard methods conceived for describing static nuclear properties; it still requires developing new methods for modelling nonequilibrium processes in nuclei, see e.g. [6]. Thus, the theoretical description of the fission process with microscopic models is still very difficult. In addition, these models suffer from their tremendous demand on computing power, restricting severely the number of degrees of freedom to be investigated.

In this situation, it is still promising to analyze the experimental findings by establishing systematic trends, by exploiting gross relations and by applying general laws of physics like the statistical model and statistical mechanics. In particular, the separability principle, an application of the macroscopicmicroscopic approach that exploits specific quantum-mechanical properties of a di-nuclear system reveals the presence of a hidden systematics that was not recognized before.

\section{Experimental systematics of fission-fragment yields}

Figure 1 gives an overview on the measured mass and nuclear-charge distributions of fission products from low-energy fission. Fission of target nuclei in the actinide region, mostly induced by neutrons, shows predominantly asymmetric mass splits. A transition to symmetric mass splits is seen around mass 258 in spontaneous fission of fusion residues.

Electromagnetic-induced fission of relativistic secondary beams covers neutron-deficient nuclei from mass 204 to mass 234 [7]. A transition from asymmetric to symmetric fission occurs around mass 226. A pronounced double-humped structure close to symmetry appears in ${ }^{201} \mathrm{Tl}$ [8] and in ${ }^{180} \mathrm{Hg}$ [9]. Also other nuclei in this mass region show similar features [10]. 


\section{Symmetric and asymmetric fission}

Bohr and Wheeler [11] explained the phenomenon of nuclear fission within the liquid-drop model by the influence of the Coulomb repulsion in heavy nuclei that favours elongated shapes and, finally, the separation into two fragments of predominantly equal size. In light nuclei, the surface energy dominates, and the conditional liquid-drop fission barrier is highest for symmetric splits, while in heavy nuclei, the Coulomb energy is decisive, and the conditional liquid-drop barrier is lowest for symmetric splits [12]. This transition occurs near $Z^{2} / A=22$ [13].

In the liquid-drop approach, the predominant asymmetric mass division in the fission of the actinides cannot be explained. Maria Goeppert-Maier [14] traced the asymmetric fission back to nuclear shell effects, in particular to the stabilizing influence of the 50 proton shell and the 82 neutron shell that coincide in the spherical doubly magic ${ }^{132} \mathrm{Sn}$. The existence of shell effects in deformed nuclei, which play an important role in nuclear fission, later evidenced by the existence of shape isomers [15] and shell-stabilized strongly deformed fragments at scission [16], was introduced by S. G. Nilsson [17].

\section{Size of the heavy fragment in asymmetric fission}

In the range where asymmetric fission prevails, e.g. from ${ }^{227} \mathrm{Ra}$ to ${ }^{256} \mathrm{Fm}$, the light and the heavy fissionproduct components gradually approach each other with increasing mass of the fissioning nucleus, see Figure 1. A quantitative analysis reveals that the mean mass of the heavy component stays approximately constant [18] at about $A=140$. This has been explained by the influence of a deformed $(\beta \approx 0.6)$ fragment shell at $N=88$ and the spherical shell at $N=82$ [16], suggesting that the position of the heavy fragment is essentially constant in neutron number.

New data on $Z$ distributions over long isotopic chains [19], however, reveal very clearly that the position in neutron number varies systematically over more than 7 units, while the position in proton number is approximately constant at $Z=54$, see Figure 2. The rather short isotopic sequences covered in former experiments did not show this feature clearly enough and gave the false impression of a constant position in mass, although indications for a constant position in $Z$ in proton-induced fission have already been deduced previously [20]. This finding represents a severe puzzle to theory, since shell-model calculations $[16,22]$ do not show any shell stabilization near $Z=54$ at a deformation of $\beta \approx 0.6$, which is suggested by the mass-dependent prompt-neutron yields, see below.

\section{Separability principle}

Ordering schemes, systematics and semi-empirical models are powerful approaches for advancing our understanding of complex phenomena in nature. In nuclear fission great progress has been made by introducing the general concept of fission channels [23]. It established a link between the observed characteristics, e.g. in fission yields and kinetic energies, and the properties of the potential-energy surface of the fissioning system. However, it did not allow for quantitative predictions. The theoretical description of nuclear fission, in particular at low excitation energies with its rich manifestation of nuclear-structure phenomena is still a challenge. At present, one is restricted to purely empirical models (e.g. [24]) for a good quantitative description of the data in their full complexity.

The microscopic-macroscopic approach has proven to be very useful for calculating nuclear properties, in particular in applications to fission [25]. The early influence of fragment shells on the fission path, revealed by two-centre shell-model calculations [26], makes its application to fission even more powerful. It means that the microscopic properties of the fissionning system are essentially determined by the shells of the fragments, and only the macroscopic properties are specific to the fissioning system [27]. 


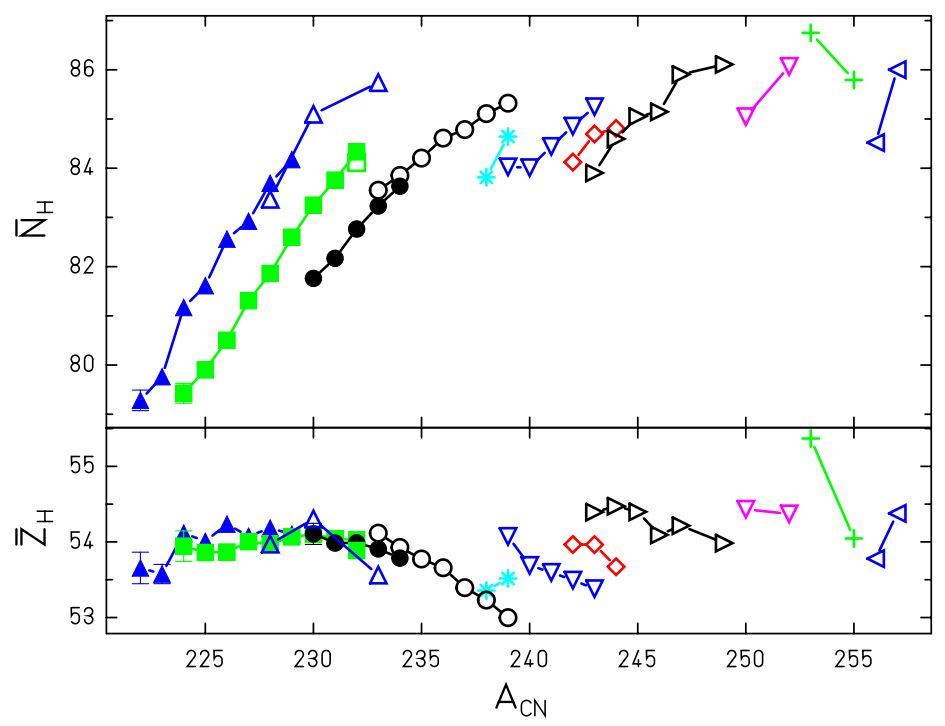

Figure 2. Mean neutron and proton number of the heavy component in asymmetric fission in the actinide region from thorium to einsteinium. The lines connect the data for a fixed element. The values were deduced from measured mass and nuclear-charge distributions using the semi-empirical GEF code [21] for the correction of charge polarization (the deviation of the $N / Z$ ratio of the primary fragments before emission of prompt neutrons from the $N / Z$ value of the fissioning nucleus) and prompt-neutron emission. Open symbols denote results from conventional experiments, full symbols refer to an experiment with relativistic projectile fragments of ${ }^{238} U$ [7]. (See $[19,21]$ for references of the underlying experimental data.)
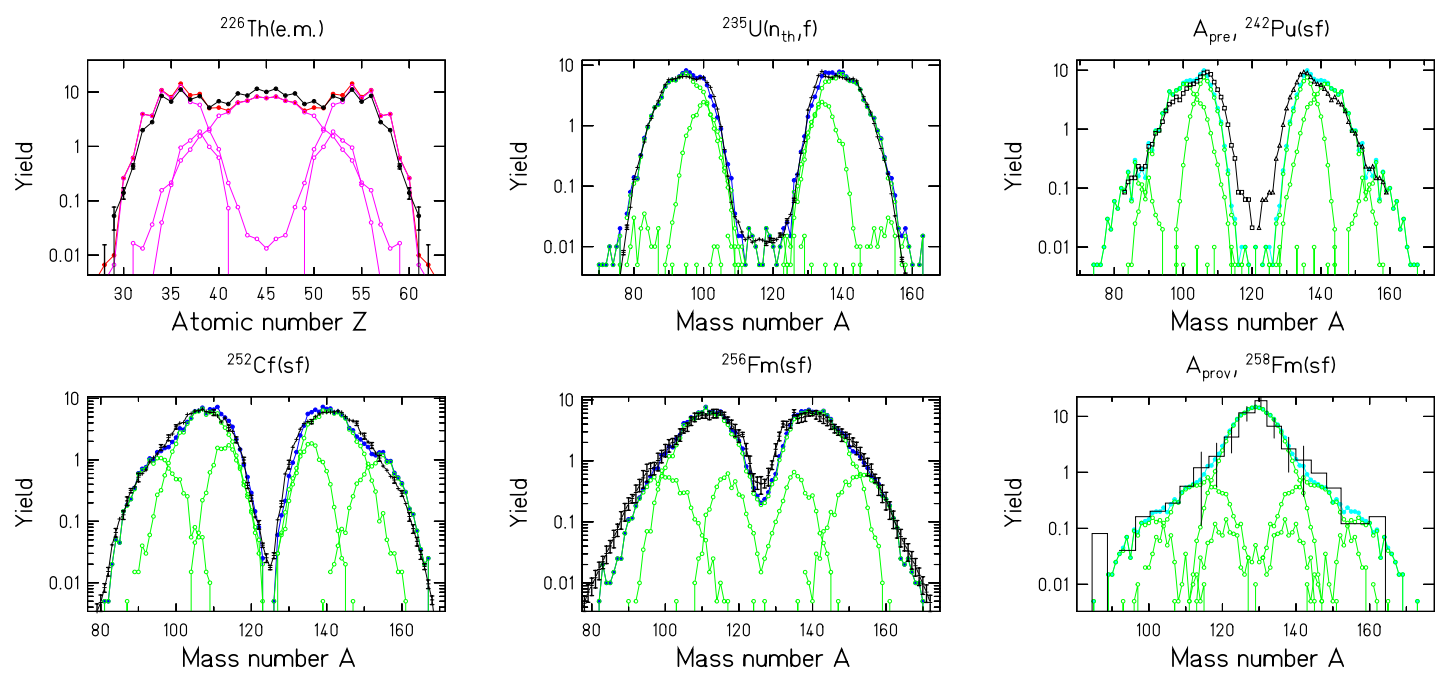

Figure 3. Mass and $Z$ distributions of fission fragments from spontaneous fission (sf), thermal-neutron-induced fission ( $\left.\mathrm{n}_{\mathrm{th}}, \mathrm{f}\right)$ and electromagnetic-induced fission (e.m.). (In most cases the post-neutron masses are shown. $A_{\text {prov }}$ is the "provisional mass" that is directly deduced from the ratio of the kinetic energies of the fragments and, thus, it is not corrected for neutron emission.) Measured or evaluated data (black lines, respectively histogram) are compared with predictions of the GEF code [21] (pink and green lines). The contributions of different fission channels are shown. (See [21] for references of the data.) 
This "separability principle" was exploited in the GEF code [21], which relies on an empirical description of the macroscopic stiffness parameters in the relevant normal modes [28] (excitations perpendicular to the fission path) and empirically deduced fragment shells. They are valid for all fissioning systems. Figure 3 demonstrates that the mass distributions over a large range of systems can be described very well with the same parameter set. The yields of the different fission channels and their mass widths are determined by the populated states of the quantum oscillators in the different fission valleys, which are assumed to be in thermal equilibrium. The properties of the nuclear heat bath are determined by the available excitation energy above the potential-energy surface and the nuclear level density. All calculations were performed with the same set of model parameters. The stiffness of the macroscopic potential against mass-asymmetric distortions was assumed to be a unique function of the fissility parameter. The function was taken from ref. [10]. The positions of the fission valleys were taken from the systematics shown in Figure 2. A unique set of the magnitudes of the shell effects that form the fission valleys and the stiffness parameters in mass-asymmetric distortions were deduced from a global fit of measured mass distributions. The variations of the fission-channel yields are caused by the interplay of the macroscopic and the microscopic features of the potential for different fissioning systems.

\section{Conclusion}

The potential-energy landscape of the fissioning systems is governed by astonishingly simple general features. Its global trends and underlying systematics are hardly directly visible in the data. The parameters that describe the population of the quantum oscillators in mass asymmetry are directly related to physical quantities, like the height of the mass-asymmetry-dependent outer potential barrier, the shell-correction energy and the properties of the normal modes beyond the outer barriers as well as the energy-dependent level-densities for the different fission channels.

This work was supported by the European Commission within the Sixth Framework Programme through EFNUDAT (project no. 036434) and within the Seventh Framework Programme through Fission-2010-ERINDA (project no. 269499).

\section{References}

[1] P. Möller, D. G. Madland, A. J. Sierk, A. Iwamoto, Nature 409, 786 (2001)

[2] T. Asano et al., J. Nucl. Radioch. Sc. 5, 1 (2004)

[3] T. Asano et al., J. Nucl. Radioch. Sc. 7, 7 (2006)

[4] J. Randrup, P. Möller, A.J. Sierk, Phys. Rev. C 84, 0334613 (2011)

[5] H. Goutte, J.F. Berger, P. Casoli, D. Gogny, Phys. Rev. C 71, 024316 (2005)

[6] A. S. Umar et al., J. Phys. G: Nucl. Part. Phys. 37, 064037 (2010)

[7] K.-H. Schmidt et al., Nucl. Phys. A 665, 221 (2000)

[8] M. G. Itkis et al., Sov. J. Nucl. Phys. 52, 601 (1990)

[9] A. N. Andreyev et al., Phys. Rev. Lett. 105, 252502 (2010)

[10] S. I. Mulgin, K.-H. Schmidt, A. Grewe, S. V. Zhdanov, Nucl. Phys. A 640, 375 (1998)

[11] N. Bohr, J. A. Wheeler, Phys. Rev. 56, 426 (1939)

[12] U. L. Businaro, S. Gallone, Nuovo Cimento 1, 1277 (1957)

[13] M. G. Itkis et al., Phys. Atom. Nucl. 58, 2026 (1995) 
[14] M. Goeppert-Mayer, Phys. Rev. 74, 235 (1948)

[15] S. M. Polikanov, Sov. Phys. USPEKHI 15, 48 (1973)

[16] B. D. Wilkins, E. P. Steinberg, R. R. Chasman, Phys. Rev. C 14, 1832 (1976)

[17] S. G. Nilsson, Kgl. Danske Videnskap Selskab, Mat. Fys. Medd. 29, 16 (1955)

[18] J. P. Unik et al., Proc. Symp. Phys. Chem. Fission, Rochester 1973 (IAEA, Vienna, 1974) vol. 2, p. 19

[19] C. Böckstiegel et al., Nucl. Phys. A 802, 12 (2008)

[20] D. M. Gorodisskiy et al., Phys. Lett. B 548, 45 (2002)

[21] K.-H. Schmidt, B. Jurado, JEF/DOC 1423 (OECD Nuclear-Energy Agency, Paris, 2012) http://www . cenbg.in2p3.fr/GEF, http://www.khs-erzhausen.de

[22] I. Ragnarsson, R. K. Sheline, Phys. Scr. 29, 385 (1984)

[23] U. Brosa, S. Grossmann, A. Müller, Phys. Rep. 197, 167 (1990)

[24] C. Wahl, Atom. Data Nucl. Data Tables 39, 1 (1988)

[25] M. Brack et al., Rev. Mod. Phys. 44, 320 (1972)

[26] U. Mosel, H. W. Schmitt, Nucl. Phys. A 165, 73 (1971)

[27] K.-H. Schmidt, A. Kelic, M. V. Ricciardi, Europh. Lett. 83, 32001 (2008)

[28] J. R. Nix, Ann. Phys. 41, 52 (1967) 